\title{
Lightning Mechanism and Atmospherics Radiation
}

\author{
By Haruzi ISIKAWA \\ (Reseach Institute of Atmospherics, Nagoya University) \\ (Read, Oct. 16, 1956 ; Received, Nov. 4, 1956)
}

Synopsis

Simultaneous recording of electro-static field change, waveform of atmospheric, lightning flash photograph, were obtained with partial success during the the thunderstorm observation in summer 1955. The simultaneous records of electrostatic field change, and waveform of atmospheric, each pair obtained at the moment of a cloud discharge, have been investigated and the possibilities have been suggested that some of the slow discharge processes in thundercloud will be mainly composed of corona discharges of various types between two charge centres in the cloud. Waveform of atmospheric, and lightning flash photograph, both relating to the same cloud discharge, have made it clear that the very rapid dart leader radiates the atmospheric with waveform of main discharge type. The directly correlated records of electro-static field change, and waveform of atmospheric, were also obtained concerning several ground discharges. This has made it possible to classify the recorded ground discharges into two types according as the discharge has $\alpha$ type stepped leader, or $\beta$ type, following to the principle orginally reported by Schonland (1). The investigation indicates that no less than $60 \%$ of the recorded ground discharges has $\beta$ type stepped leaders in this thunderstorm season. In spite of the insufficient number of available data at present it seems reasonable to consider that the greater part of the ground discharges in our country have $\beta$ type stepped leaders.

It has also been made clear that the streamer emitting an outstanding single pulse, such as the return streamer of ground discharge, is always associated with secondary and somewhat slow discharge processes.

\section{Introduction}

There are great many varieties among lightning dicharges and they change their appearances not only from a thunderstorm to another but also from discharges occurring at a certain stage of thunderstorm to those at another stage of the same storm. Corresopondingly the waveforms of atmospherics radiating from these lightning discharges are very diverse and in addition much complicated. The discharges occurring at slow rates in thunderclouds are esspecially complex. It remains here many points unknown concerning the mechanisms of cloud discharges, because the luminous phenomena associated with discharges in thundercloud can very hardly be photographed. To investigate these points more clearly, another new amplifier channel of superheterodyne type with the tuning frequency $10 \mathrm{Mc} / \mathrm{sec}$. has been attached to the previous waveform recorder [2]. This channel simultaneously triggers two recording C.R.T.'s 
which give the wasveform of atmospheric and the static field change on respective fluorescent screens. The image reproduced on these two C.R.T. screens are photographed in respective frames on $35 \mathrm{~mm}$ film by respective cameras. This improvement of the apparatus has made is possible to record the variation of electro-magnetic field due to lightning discharge to ground from leader to return, which was very difficult with the previous one. Accordingly it has also become possible to compare the waveform, and the static field change, of a lightning discharge with the resolved photograph of the same lightning flash and to analyse the correlation between discharge mechanism and radiation of atmospheric.

\section{Investigation of the observational facts}

1. Cloud discharges producing slow electro-static field changes.

Two examples of typical records, each obtained at the moment of a cloud discharge, are illustrated in Fig. 1. and 2. In these figures, A represents the electro-static field change, and $\mathrm{B}$ the corresponding wave-form of atmospheric, each pair produced by the same cloud discharge. These two illustrate the case where the cloud discharge produces the slow static field change, which shall be denoted as "S-process" in what follows. It is now generally recognized among research workers in the

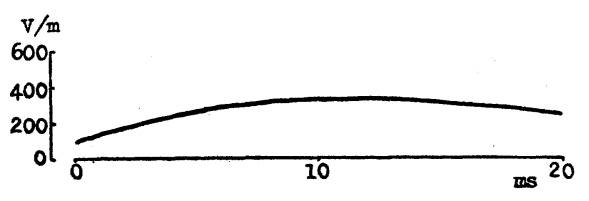

A

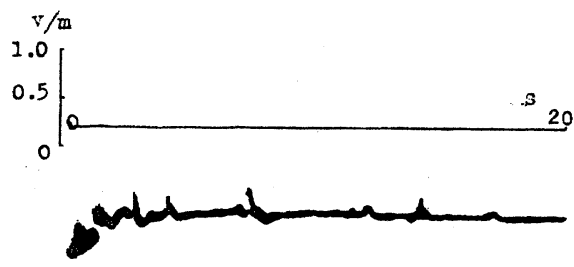

B

Fig. 1. Cloud discharge producing slow electro-static field change and grouped radiatian pulses.

A. Slow electro-static field change.

B. Waveform of atmospheric with grouped pulses.

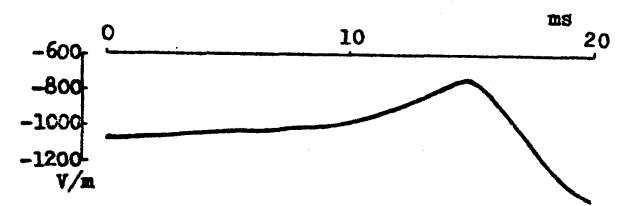

A

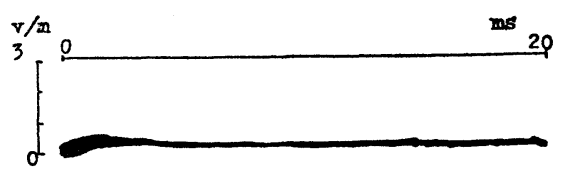

B

Fig. 2. Cloud discharage producing slow electro-static field change and isolated radiation pulses.

A. Slow electro-static field change.

B. Waveform of atmospheric with isolated pulses.

world that the greater part of cloud discharges are composed of these S-processes. Simultaneous and continuous method of recording the waveform of atmospheric, and the static field change, performed in our country concerning cloud discharges [3], revealed the fact that radiation pulses present themselves at mean time intervals 8-16 m-sec. in the course of a S-process, taking the form of groups with mean 
duration of several $\mathrm{m}$-sec. in one case, and the form of isolated pulses in another. In contrast to this continuous method of recording, the present apparatus picks up the phenomena in the period of $20 \mathrm{~m}$-sec. after the recording has been triggered by a pulse of atmospheric, and hence it catches the moment of a cloud discharge when a certain group of pulses is coming about. Fig. 1 shows the case where S-process emits groups of pulses intermittently, and Fig. 2. the case where the process emits isolated pulses at time intervals roughly no less than $20 \mathrm{~m}$-sec. Table 1. represents the number of recorded waveforms of grouped pulse type, and the isolated pulse type, both radiating from cloud discharges, and indicates that the occurrence probabilities of these two are nearly the same. To investigate the differences of time rates of slow static field changes between these two types, the mean time rate has been measured for each case employing 18 records of static field changes, produced by the processes occurring at distances in the range $8-10 \mathrm{~km}$. from the station, and included both these two types.

Table 1. Number of recroded waveforms of grouped pulse type, and isolated pulse type, referring to cloud discharges.

\begin{tabular}{|l|c|c|}
\hline & Grouped pulse type & Isolated pulse type \\
\hline $\begin{array}{l}\text { Number of recorded } \\
\text { waveforms }\end{array}$ & 71 & 70 \\
\hline
\end{tabular}

The result is shown in Table 2., which seems to indicate that although by cloud discharges emitting grouped pulses can the electricity be transferred a little faster than by those emitting pulses. scatteredly, it is unlikely to consider any significant differences lying between these two. This can be interpreted by the fact that the amplitude of a radiation pulse is not determined by the quantity of electricity transferred by the rapid streamer process related to it along a discharge channel, but mainly determined by the

Table 2. Time rates of electro-static field changes produced by S-processes.

\begin{tabular}{|c|c|c|}
\hline Distance & Grouped pulse type & Isolated pulse type \\
\hline $8-10 \mathrm{~km}$. & $9.4 \mathrm{v} / \mathrm{m} . \mathrm{m}$-sec. & $7.5 \mathrm{v} / \mathrm{m} . \mathrm{m}-\mathrm{sec}$. \\
\hline
\end{tabular}

time rate of electric current variation along it, and the discharge streamers emitting radiation pulses do not seriously contribute to transfer the electricity. However these streamer discharges may contribute, though only a little, to the transfer of electricity, if they come into existence intermittently forming large groups of pulses in the course of a cloud discharge. This seems to interpret the slight difference of the time rates of slow field changes between grouped pulse type, and isolated pulse type, as indicated in Table 2. The greater part of electricity contributing to a cloud discharge therefore must be transferred, and canceled out, by other discharge mechanisms with slow and continuous character. These mechanisms of cloud discharges are of course very different from those of rapid streamer discharges emitting radiation pulses. As individual radia. tion pulses appearing in each group on the waveform of S-process present themselves 
neraly at random [4], it seems reasonable to censider that during the course of $\mathrm{S}$. process, random but rapid streamers on small scales present themselves in grouped form in one case and in isolated form in another. S-process may be considered to have the very similar nature to that of J-process occurring in the intervals between strokes of multiple ground discharges [5], F-process often occurring at the final stage of less multiple ground discharges [6], and the continuting stroke follow. ing upward stepped leaders [7]. However only a little has been known about the mechanisms of S-processes up to the present. If S-process taking place in thundercloud has the same machanism as J-process has, it must be composed of the positive streamer that results in the transfer of positive electricity in the cloud. Allibone and Meek [8] made experimental studies on the development of long spark discharge and showed that the velocities of leader streamers increase with discharge gaplength independent of their polarities, if all other conditions are the same. An example of their results is shown in Table 3 . If the relation holds good from

Table 3. Variation of leader velocities with gap-lengths in experimental spark discharges.

\begin{tabular}{|c|c|c|c|}
\hline Polarity & $\begin{array}{c}\text { Discharge gap-length } \\
\text { (mm.) }\end{array}$ & $\begin{array}{c}\text { Velocity of leader } \\
\text { streamer (cm/sce) }\end{array}$ & $\begin{array}{c}\text { Over voltage } \\
(\%)\end{array}$ \\
\hline- & 25 & $6 \times 10^{6}$ & 0 \\
- & 150 & $17 \times 10^{6}$ & 0 \\
\hline+ & 25 & $4 \times 10^{6}$ & 0 \\
+ & 150 & $15 \times 10^{6}$ & 0 \\
\hline
\end{tabular}

experimental spark discharges on small scales to lightning discharges on huge scales, the negative pilot streamer appearing in the first leader of ground discharge must have larger velocity than what is represented in Table 3., as the extreme case of long spark discharges. The observed velocity values of pilot streamers in discharges to ground are reported to be in the range $1-8 \times 10^{7} \mathrm{~cm} / \mathrm{sec}$. [9], which seems to support this point. Being considered from the theoretical point of view, the positive leader streamers, as Allibone and Meek indicates [8], must have velocitis larger than those of negative one, which is really supported by the results of experimental studies on spark discharge development [8]. An example of them is illustreated in Table 4. If the arguments can be applied to lightning leader streamers developing in unionized atmosphere, the velocities of positive leader streamers must be larger than those of negative ones. Therefore the velocities of the formers developing in the virgin atmosphere can be estimated to be in the range $3-16 \times 10^{7} \mathrm{~cm} / \mathrm{sec}$. under the assumption that the relation

Table 4. Variation of leader velocities with gap lengths in experimental spark discharges.

\begin{tabular}{|c|c|c|c|}
\hline \begin{tabular}{c} 
Polarity \\
\hline+
\end{tabular} & $\begin{array}{c}\text { Discharge gap-length } \\
(\mathrm{mm})\end{array}$ & $\begin{array}{c}\text { Velocity of leader } \\
\text { streames }(\mathrm{cm} / \mathrm{sec})\end{array}$ & $\begin{array}{c}\text { Over voltage } \\
(\%)\end{array}$ \\
\hline- & 71 & $2.6 \times 10^{6}$ & 0 \\
\hline
\end{tabular}

indicated in Table 4 . is applicable to the case of lightning discharges. The actual velocity of J-process streamer was originally estimated by Malan and Schonland [5] 
to be about $3 \times 10^{6} \mathrm{~cm} / \mathrm{sec}$. from the investigation of slow electro-static field changes occurring in the intervals between strokes of gound discharges, under the assumption that the positive streamer develops upward in the negatively charged column in thundercloud during periods of stroke intervals, and later this was more directly determined by Hewitt [10] from time interval measurements of radar echoes from the channel of ground discharge. Hewitt sometimes observed that the time interval between the direct pulse, and the echo pulse that was assumed to come from J-streamer channel, increased slightly with time in steps from one stroke to the next, and estimated the vertical velocities of the assumed J-streamer at $0.4-1 \times 10^{6} \mathrm{~cm} / \mathrm{sec}$. F-process, as reported by Malan [6], may also be considered to be a kind of J-process that often occurs after the last stroke of a multiple discharge to ground with roughly less than 4 strokes, and the velocity of it was estimated by him at about $2.5 \times 10^{6} \mathrm{~cm} / \mathrm{sec}$. This seems not to agree with the results deduced from experimental studies on long spark discharge development. The reason for this may be the following two: first, in the atmosphere, such as the interior of thundercloud, does the positive streamer develop slower than it does in the experimental spark gap; and second, the slow and continuous discharge in thundercloud involves not only discontinuous rapid streamer discharges, but also continuous slow corona discharges at the same time, and indeed, it often happens that the former occupies only a small part of a cloud discharge and the latter occupies the greater part of it. Both of these two may be considered to occur often simultaneously in thunderclouds, but which of these occurs more frequenly and is more probable than the other, it is a matter of question at present.

The discharges in thunderclouds can very often be reduced to the model that is composed of a discharge between two change centres [11] with spherical forms, one of which is positive and lies in the upper part of a thundercloud, and the other is negative and lies in the lower part of it. The radius of spherical regions constructing these two charge centres, and carrying the electricity contributing to a lightning discharge is estimated to be about $0.1 \mathrm{~km}$. [12]. When the potential gradient round these two assumed charge centres, the separation of which is estimate at about $5 \mathrm{~km}$., reaches to the critical value necessary for the onset of corona discharges, the corona will come about along the surface of inward hemispheres of the two opposing charge centres. The dark current region that connects these two corona regions, and carries the discharge current, will have a columnar form, the radius of which will of course vary from one position to another between these two charge centres. As the corona current density through the electrode surface, computed from the experimental corana discharge between two parallel wires [13], is about $1.3 \times 10^{-7} \mathrm{amp} / \mathrm{cm}^{2}$, the corona current of the cloud discharge will be estimated at about 80 amp., under the assumption that the current with this density flows out through hemispherical surfaces of the two charge centres. This is in good agreement with the average value of the order $10^{2} \mathrm{amp}$. of current of S-processe in thundercloud, which has been computed under the assumption that one S-process in the cloud discharges 20 coul. in $0.2 \mathrm{sec}$. on the average; this seems plausible enough, if we consider the observational facts about discharges in 
the cloud. Following to the above discussions, it is possible to infer the coronas as well as slow positive streamers existing in thunderclouds. The corona type discharges in the cloud necessarily has the continuous character and can more readily develop from positive charge centre than negative one, because this type is mainly composed of corona processes between two equally opposing charge centres that lie in the upper positive region of the cloud and the lower negative region of it respectively. The non-uniform distribution of electricity within each centre, and the irregularities in electric field around it, probably, accelerate the production of intermittent streamers on small scales, such as brush corona, and streamer corona, which can be observed in experimental corona discharges. This seems to explain the observational fact that the random radiation pulses assumedly related to streamer discharges occur in the coures of a slow continuous discharge in the cloud. Because in the actual cell of a thundercloud the electro-static field scarcely exceeds $3 \times 10^{3} \mathrm{v} / \mathrm{cm}$ [14], and any appreciable positive space charge triggering a cloud discharge can hardly be considered to exist in the interior of the cloud, in contrast to the case of discharge to ground; the greater part of cloud discharges must be initiated by these corona processes. The positive corona generally develops more readily than negative one, as experiments indicate, hence the random corona streamers that occasionaly occur in the course of a slow cloud discharge, depending on conditions round two charge centres, must mainly have positive polarity. The velocities of positive streamers estimated from the results of experimental spark discharges are roughly $10^{8} \mathrm{~cm} / \mathrm{sec}$. [15], and the same order of magnitude as those of dart leaders, therefore the widths of radiation pulses appearing in the course of a cloud discharge seems to be interpreted by positive corona streamers, as well as negative dart leaders [16].

2. Cloud discharges accompanying step field changes.

Some of the cloud discharges produce rapid changes of electro-static field, which will be denoted as "R-process" in the followings, and an example of which is illustrated in Fig. 3. In the figure, A shows a step field change, and B a clear single radiation pulse, both produced by the same $\mathrm{R}$-process. This seems to suggest that the cloud discharge process producing a fairly outstanding step field change is mainly composed of rapid streamer emitting a clear radiation pulse. This kind of rapid streamer can, in some cases, be considered as being the special dart leader, because the leader is the only kind of rapid streamer process that has been observed in nonground discharges. This will be illustrated just further on. In Fig. 3., A indicates that the step field change is produced by R-process in $3 \mathrm{~m}$-sec., and $\mathrm{B}$ the corresponding radiation pulse being emitted in only $1.6 \mathrm{~m}$-sec. It can be concluded from these facts that even the special dart leader streamer, occurring in the course of a cloud discharge, and emitting single radiation pulse, always accompanies secondary discharges with somewhat slow time rates. This will also be discussed later on again. Fig. 4. illustrates the case where the rapid dart leader emits the outstanding radiation pulse of main discharge type. A shows the cloud flash, photographed by Boys' camera, and composed of single dart leader, and $\mathrm{B}$ the radiation pulse emitted by this cloud dis- 

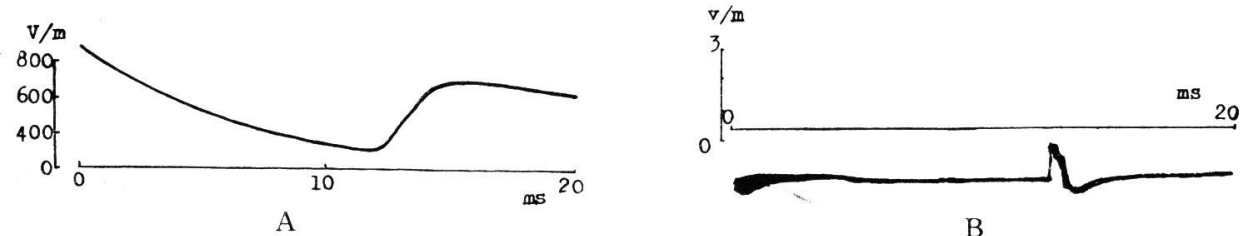

Fig. 3. Cloud discharge accompanying step field change.

A. Step electro-static field change.

B. Radiation pulse corresponding to $\Lambda$.
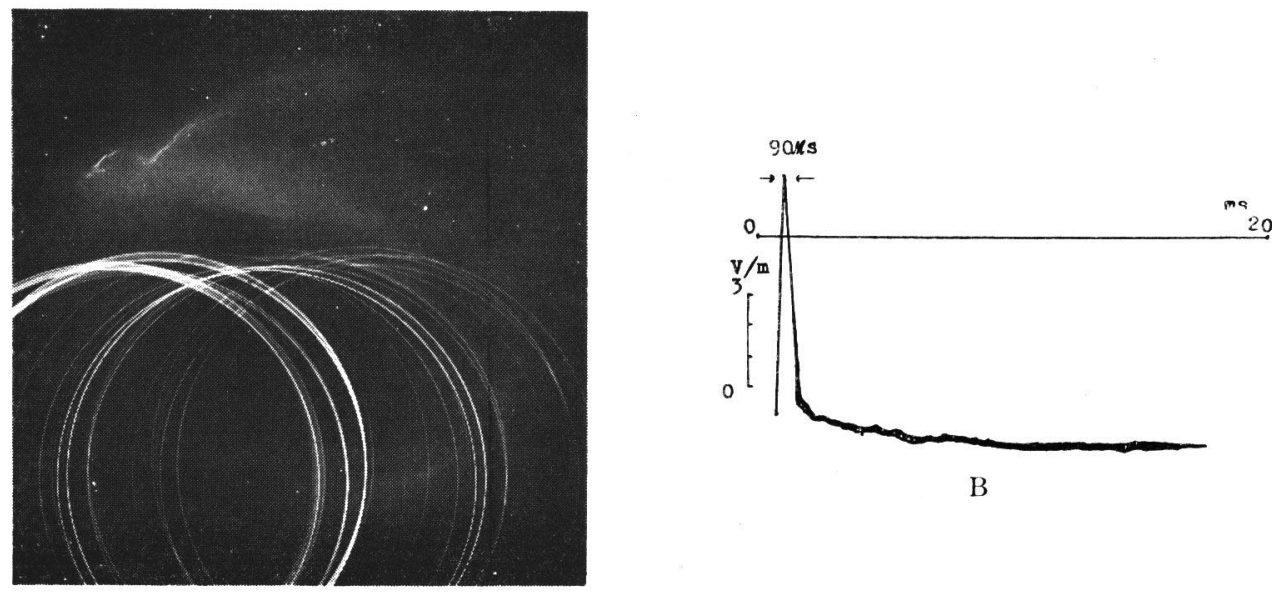

B

Fig. 4. Cloud discharge emitting main discharge type waveform.

A. Cloud flash photographed by Boys' camera.

B. Main discharge type waveform corresponding to A.

charge. As the waveform illustrated in Fig. 4. B has the very similar structure to that of main discharge type, it may be reasonable to consider that this waveform is emitted by the dart leader illustrated in A. It is evident, on the other hand, from the visual observation that this cloud discharge was photographed at the distance about $10 \mathrm{~km}$. from the discharging point, therefore the total channel length of the flash can be estimated at about $4 \mathrm{~km}$. by measuring the length of image of the flash on photographic plate. The width of the corresponding pulse of the waveform can also be estimated at about 100 micro-sec. on the record $\mathrm{B}$, so that the advancing velocity of this dart leader is roughly $4 \times 10^{9} \mathrm{~cm} / \mathrm{sec}$. This is much larger than $0.1-2.3 \times 10^{9} \mathrm{~cm} /$ sec., the velocity values of the dart leaders obtained by Schonland and Malan [17]. Therefore it is evident that such rapid dart leader, as the photograph illustrates, produces an outstanding field change and emits a clear radiation pulse of the similar nature to that of the main discharge type waveform. This agrees with our conclusions already deduced from the investigation of the occurrence frequency of waveforms of this type [16]. In Fig. 5., A illustrates the photograph of a cloud flash composed of six dart leaders, and B the waveform of atmospheric which was recorded simultaneously with the photograph A. As the figure indicates, the waveform is composed of random pulses forming a group with duration larger than $2 \mathrm{~m}$-sec. at least. It has 
no such outstanding radiation pulse that can be interpreted as being emitted by one of these six dart leaders, and is similar to the return streamer pulse illustrated in Fig. 6. Following to the discussions in Section 1., it will be probable to conclude that the
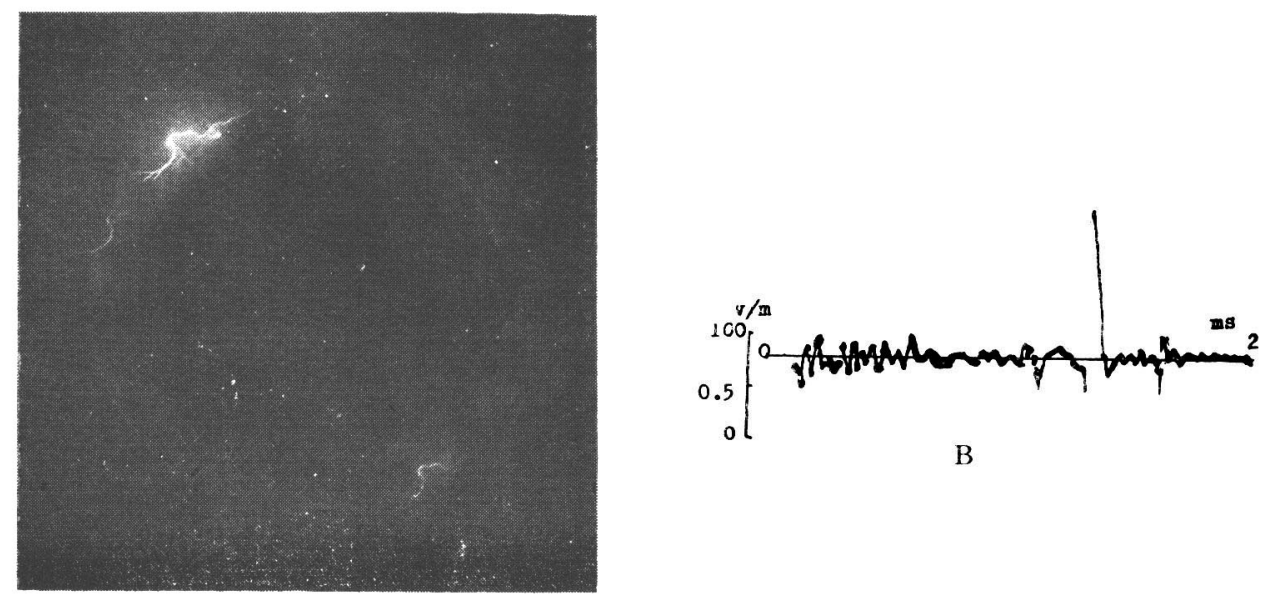

A

Fig. 5. Multiple cloud discharge emitting grouped radiation pulses.

A. Photograph of multiple cloud flash obtained by Boys' camera (sixfold discharge)

B. Waveform of grouped radiation pulses corresponding to A.

waveform of grouped pulses in Fig. 5. B is produced by small but rapid streamers, occurring at random in the course of slow discharge processes, and preceding these six dart leaders.

3. Discharge to ground.

Fig. 6. illustrates an example of the records of discharge to ground, and A shows the rapid step field change and $\mathrm{B}$ the outstanding single radiation pulse, both being produced by the same return stroke of discharge to ground in the vicinity. The wave-

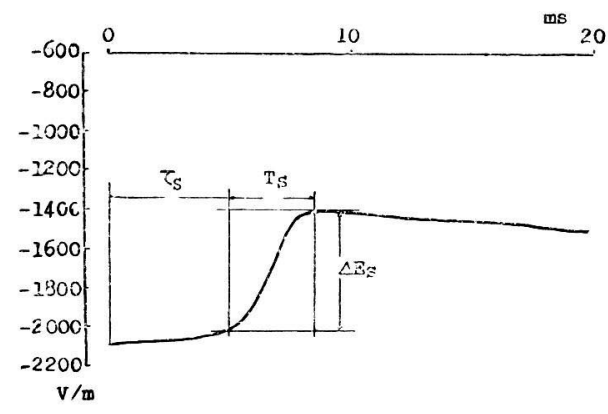

A

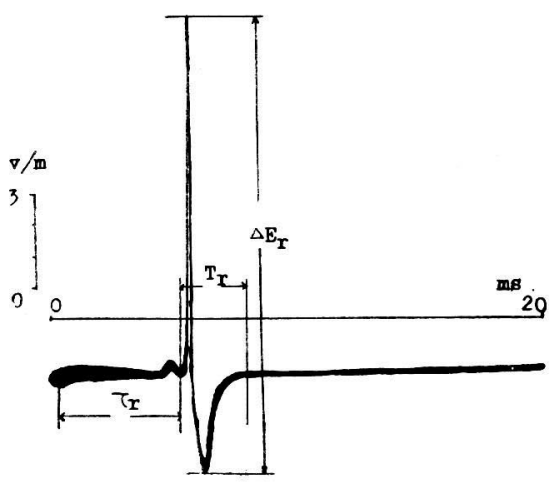

B

Fig. 6. Record of ground discharge.

$\Lambda$. Rapid step field change.

B. Waveform of atmospheric corresponding to A.

form $\mathrm{B}$ was obtained by using the waveform recorder with the frequency response chara- 
cteristics, for which the low frequency side was cut off at $0.5 \mathrm{kc} / \mathrm{sec}$. in order to not to record the static field component of electro-magnetic field variation, but to emphasis the radiation component of it, hence such waveforms as the figure illustrates, must fairly be distorted by these instrumental causes, and does not, of course, represent the radiation component strictly. The measurement of pulse width, $\mathrm{Tr}$, of the waveform $B$ gives the value of about $3.2 \mathrm{~m}-\mathrm{sec}$, which is much larger than the duration of luminosity, i.e., several hundred microseconds at the largest at the channel root of the ground discharge photographed by the authors [16]; so it is evident that the pulse width of the waveform of ground discharge caught by the present apparatus is generally larger than the duration of luminosity at the channel root of the relating flash, which gives the direct indication of the duration of return stroke and hence determines the true radiation pulse width on the wavform [16]. The rapid step field change of this discharge is performed in $4.3 \mathrm{~m}$-sec., as the figure illustrates, so it is recognized here again that the width of radiation pulse relating to return stroke is larger than the duration of the corresponding step field change, which is the same relation as the rapid cloud discharge discussed in Section 2 has indicated already. To clear out these points further-more the starting time, $\tau_{s}, \tau_{r}$, of step field change and relating radiation pulse, were measured with regard to ground discharges, and the results are summarized in Table 5. It is clear from the table that the step field change produced by return stroke starts in every case a little earlier than radiation pulse emitted by the same stroke. This indicates that the step field change that roughly relates to a return stroke includes not only the part produced by return streamer itself but also, even a little, the part produced by the final part of the concerning leader stroke [1].

Table 5. The comparison of starting time of step field change, with that of radiation pulse, both referring to the same ground discharge.

\begin{tabular}{|c|c|c|c|}
\hline Sign of $\tau_{s}-\tau_{r}$ & + & 0 & - \\
\hline Frequency of occurrence in $\%$ & 0 & 55 & 45 \\
\hline
\end{tabular}

The duration, $T_{s}$, of the step field change produced by return stroke, and the width, $T_{r}$, of the radiation pulse corresponding to it are measured on each pair of records of ground discharges. The percentages of the number of records that have the same sign of the value, $T_{s}-T_{r}$, are tabulated in Table 6 ., which shows that there is no cases where $T_{r}$ is clearly larger than $T_{s}$.

Table 6. The comparison of duration of step field change with width of outstanding radiation pulse, both produced by the same ground discharge.

\begin{tabular}{|c|c|c|c|}
\hline Sign of $\mathrm{T}_{s}-\mathrm{T}_{r}$ & + & 0 & - \\
\hline Frequency of occurrence in $\%$ & 61 & 39 & 0 \\
\hline
\end{tabular}

Therefore in spite of the facts that the waveforms are fairly distorted and the static field changes were recorded not sufficiently clearly, it is reasonable to conclude that the return streamer producing clear single radiation pulse does not responsible for the 
whole of a step field change, but the latter is always accompanied by sowewhat slow secondary discharges correlated to the return stroke, such as the final part of concerning first leader, and the "c" portion of ground discharge or the beginning part of the following J-process. Most of the records of radiation pulses related to return strokes have the leader parts, and some of them carry many radiation pulses on their leader sections at intervals similar to those between step streamers of the first leader of ground discharge, and the others do not carry any such pulses on it. As the waveform of ground discharge recorded by the present apparatus refer to the first stroke of a ground discharge, the above difference about leader sections of the recorded waveforms must be attributed to the nature of the first leaders, i.e., the pulsive leader waveform must relate to $\alpha$ type stepped leader and the non-pulsive one to $\beta$ type [1]. In all, 17 records of ground discharge waveforms are available for the discussion, which were caught from leader section to return stroke by the recorder adjusted to the same sensitivity. These records have been classified into three cases: $\alpha$ type, $\beta$ type, and unclassifiable. The results are given in Table 7 . The table seems to indicates that the greater part of the ground discharges occurred in this period of thunderstorm observation accompanied $\beta$ type first leaders [18]. The statistical investigations previously

Table 7. The classification of the first leaders of discharges to ground.

\begin{tabular}{|c|c|c|c|}
\hline Type of first leaders & $\alpha$ type & $\beta$ type & unclassifiable \\
\hline No. of recorded waveforms & 2 & 10 & 5 \\
\hline
\end{tabular}

made by the authors about cloud discharge :waveforms recorded through past 3 summers [4], made it clear that many of the discharges in the cloud, if they involve something like stepped leaders, mostly accompany $\beta$ type leaders. Hence, generally speaking, it is plausible to conclude that the greater part of stepped leaders occurring in our country are of $\beta$ type, indifferent to the fact that they relate to ground discharges or to cloud discharges.

\section{Conclusion}

It can be concluded from the above discussions that:

(1) Most of the cloud discharges are composed of slow and continuous discharge processes. It seems possible, in some cases, to interpret these processes by the discharge model, the main part of which is composed of coronas between two spherical charge centres, one of which is in the upper positive part of thundercloud and the other in the lower negative part of it.

(2) In the course of a slow cloud discharge the radiation pulses are emitted by random rapid streamers. These radiation pulses are produced in groups and form many intermittent bursts in some cases, and they form no more than the isolated random pulses in others.

(3) Some of cloud discharges produce special dart leaders with very high velocities, and the radiation pulses of main discharge type are emitted by them in such cases. 
(4) In general, the rapid and large streamer emitting an outstanding radiation pulse, always accompanies secondary slow discharge processes related to the streamer.

(5) So long as our observation concerns, it is reasonable to consider that most of the stepped leaders occurring in our country are classified to $\beta$ type, indifferent to the fact that they relate to cloud discharges or to ground discharges.

In conclusion authors' sincere thanks are due to Prof. A. Kimpara, the Director of our Institute, who has encouraged them throughout the present investigation. The data employed to the present investigations were obtained by using the apparatus that had been constructed by him under the aid of a Grant in Aid of the Miscellaneous Scientific Research from the Ministry of Education.

\section{References}

[1] Schonland B.F.J. et al. : Proc. Roy. Soc. A, 166, 56 (1938)

[2] Authors: Proc. Res. Inst. Atmosph. 1, 12 (1953)

[3] Kitagawa S.: Data for the Report of Thund. Res. Committ. Power and Weather Coord. Committ. (1955) (In Japanese)

[4] Authors: Proc. Res. Inst. Atmosph. 3, 29 (1955)

[5] Malan D.J. et al. : Proc. Roy. Soc. A, 206, 145 (1951)

[6] Malan D.J. : Ann. d. Geophys. 10, 271 (1954)

[7] McEachron K.B. : J. Frank. Inst. 277, 149 (1939)

[8] Allibone T.E. et al.: Proc. Roy. Soc. A, 166, 97 (1938)

[9] Schonland B.F.J. : Hand. d. Phys. Bd. 22 (1956)

[10] Hewitt F.J. : Proc. Phys. Soc. B, 66, 895 (1953)

[11] Workman E.J. et al.: Tech. Not. Nat. Advis. Committ. Aeron. No. 864, Nov. (1942)

[12] Honda K. : Mag. Thund. Res. 1, No. 8, 23 (1953) (In Japanese)

[13] I.E.E. Japan : Discharge Phenomena 166 (1952) (In Japanese)

[14] Norinder H.: Arkiv. Geofys. 2, 97 (1954)

[15] Loeb L.B. et al.: The Mechanism of Electric Spark 38 (1941)

[16] Authors: Proc. Res. Inst. Atmosph. 2, 9 (1954)

[17] Schonland B.F.J. et al. : Proc. Roy. Soc. A, 152, 595 (1935)

[18] Kitagawa S. : Mag. Thund. Res. 3, No. 6, 23 (1955) (In Japanese) 